\title{
An Opinion on Routine Rabies Pre-Exposure Prophylaxis for Children in High Endemic Areas \\ Hildegund CJ ErtI
}

The Wistar Institute, 360 Spruce Street, Philadelphia, PA 19104, USA

"Corresponding author: The Wistar Institute, 360 Spruce Street, Philadelphia, PA 19104, USA, Tel: 2158983863; E-mail: ertl@wistar.org

Received date: December 12, 2017; Accepted date: December 18, 2017; Published date: December 25, 2017

Copyright: @2017 Ertl HCJ. This is an open-access article distributed under the terms of the Creative Commons Attribution License, which permits unrestricted use, distribution, and reproduction in any medium, provided the original author and source are credited.

\section{Opinion}

Rabies virus continues to cause more than 50,000 reported human deaths each year. The actual number is likely to be higher. Nearly half of the victims are children below the age of 15 [1]. In most case rabies virus is transmitted through bites or licks by infected dogs. Rabies virus is a simple bullet shaped virus with a single stranded RNA genome. The virus, once transmitted, travels within axons through retrograde transport to the central nervous system, where is replicates and causes encephalitis, which, once symptoms develop, is nearly always fatal. During the late stages of infection, the virus travels back to the periphery and is shed in saliva from where it is transmitted. It can also be found in other tissues and the use of organs from rabies victims has led to transmission to transplant recipients [2]. The incubation time of rabies virus is highly variable ranging from a few days to several years. During long incubation periods, it is unknown where the virus may hide.

Safe and efficacious rabies vaccines are available. They are based on inactivated virus and mediate protection through induction of rabies virus neutralizing antibodies directed against the viral glycoprotein. As infections are memorable, vaccines are in general given after exposure to a rabid animal and only individuals at very high risk are vaccinated preventatively. In addition to thorough wound cleaning, post-exposure prophylaxis (PEP) involves several doses of vaccine. In cases of severe exposure, the first vaccine dose has to be combined with a rabies immune serum (RIG) that should largely be injected into the site of exposure. PEP, which must be initiated shortly after exposure, is very costly not only in terms of biologicals but also travel and loss of time. In developing countries this together with ignorance of the consequences of exposure to a rabid animal and lack of access to health care facilities or the needed biologicals, especially RIG, is linked to lack of needed vaccinations and consequently death due to rabies.

Other vaccines, which are routinely given to children as part of the Expanded Program of Immunization (EPI), prevent viral diseases that cause far lower fatality rates. For example, in the United States virus infections such as Mumps and Rubella each were linked to less than 100 fatalities annually before vaccines became available [3].

Rabies pre-exposure vaccination (PrEP) of children is safe and effective as was demonstrated in Peru where now in highly endemic areas, the rabies vaccine is included into the childhood immunization program. Peru has been suffering periodically from increased rates of rabies death in the Amazon jungle due to transmission from infected vampire bats [4]. Health care in Amazonia relays on a few health care centers that for many residents are only accessible by 2-3 day trips by canoes or by long walks through the forest. Other areas are served by Medical Boats that visit each community in weekly or 2-weekly intervals. Rapid medical interventions are thus unavailable to most residents. Many lack basic knowledge on infectious diseases and prefer traditional to modern medicine. Village Elders still blame rabies on witchcraft [5]. Avoidance of exposure is impossible; most residents of the Peruvian jungle sleep in open-air dwellings that can easily be entered by bats. Control of rabies in vampire bats through their vaccination is currently not possible [6] and culling of bats although practiced is not effective and may actually cause harm by increasing numbers of juvenile bats that are the most common transmitters of rabies virus [7]. During a rabies outbreak in 2011 Peru started a mass rabies vaccination program in remote providences that were deemed at high-risk for rabies. The incidence of rabies declined dramatically in these areas and only none of the vaccinated individuals became infected.

Similarly in the Philippines where rabies virus is mainly transmitted by dogs, the government implemented in 2007 free routine rabies PrEP for school children aged 5-15 living in highly endemic areas. Unlike in Peru vaccines were given intradermally, which allows for dose sparing and thereby reduces the cost of vaccination. The program was stopped in 2011 due to vaccine shortage. The World Health Organization and governments of other countries recommend childhood rabies vaccination but without access to free vaccines such recommendations are rarely followed [8].

A number of studies assessed the cost effectiveness of routine rabies PrEP of children using current vaccines. Even upon PrEP, individuals must receive PEP upon documented exposure although they only need two boosters, or as must be further explored, potentially only one booster immunization, and do not require RIG. This must be included in cost estimates. Also, rabies vaccination could be combined with other childhood vaccines, which reduces expenses due to travel and loss of wages. A Thai study estimated that taking all of this into consideration childhood PrEP by intradermal vaccination would be cost neutral if the annual risk of exposure exceeded $20 \%$. As in most areas including in Thailand the risk of exposure is markedly lower at around $2-3 \%$, it was concluded that rabies PEP was not cost effective [9]. Rabies vaccines that would cost no more than $\$ 1.32$ would achieve cost neutrality for routine PrEP if $\sim 1 \%$ of children were exposed each year to rabies [10].

An ideal vaccine for routine childhood rabies PrEP would induce sustained protective titers of neutralizing antibodies after a single dose. It would induce a potent memory B cell response that could be rapidly recalled by a single dose of a traditional rabies vaccine in case of exposure to a rabid animal. The vaccine would be effective and safe in small children between the ages of 1-2 years and it could be combined with other vaccines that are part of the EPI. As already mentioned, its cost could not exceed $\$ 1.32$ per dose. Such a vaccine is currently not available but two vaccines that have undergone pre-clinical testing potentially meet these requirements. One is a genetically modified attenuated rabies vaccine that expresses two copies of the viral 
Citation: Ertl HCJ (2018) An Opinion on Routine Rabies Pre-Exposure Prophylaxis for Children in High Endemic Areas. J Vaccines Vaccin 9: 379. doi:10.4172/2157-7560.1000379

Page 2 of 2

glycoprotein, which is the target of virus neutralizing antibodies. Due to higher expression of the glycoprotein the vaccine induces in experimental animals rapid protection after a single dose [11]. It is currently unknown if this vaccine, which is attenuated rather than inactivated, would be acceptable for use in children and if vaccine production could be scaled up to meet global needs and if the vaccine would be significantly cheaper than current vaccines. Another vaccine that my group developed is based on a replication-defective adenovirus vector derived from a chimpanzee isolate expressing the rabies virus glycoprotein. Most humans lack neutralizing antibodies to chimpanzee adenoviruses, which could blunt the rabies virus-specific antibody response [12]. In animals, including nonhuman primates, a single moderate dose of the recombinant subunit vaccine induces high titers of rabies neutralizing antibodies, memory $\mathrm{B}$ cells and most importantly, complete protection against a severe challenge with a virulent strain of rabies virus [13]. Production, purification and release testing procedures for recombinant replication-defective adenoviruses are well established. Cost-estimates show that the vaccine, once massproduced, could cost as little as $\$ 1$ [14]. The vaccine like all genetic vaccines that rely on in situ production of the antigen, which causes a delay in the onset of immune responses, is not suited for PEP where rapid induction of protective antibody titers before the virus establishes an infection in the central nervous system is of the essence. Nevertheless, it is a suitable candidate for childhood PEP and at this stage should be explored in human trials.

\section{References}

1. Hampson K, Coudeville L, Lembo T, Sambo M, Kieffer A, et al. (2015) Global Alliance for Rabies Control Partners for Rabies Prevention. Estimating the global burden of endemic canine rabies. PLoS Negl Trop Dis 9: $\mathrm{e} 0003709$.

2. Vora NM, Basavaraju SV, Feldman KA, Paddock CD, Orciari L, et al. (2013) Transplant-Associated Rabies Virus Transmission Investigation Team. Raccoon rabies virus variant transmission through solid organ transplantation. JAMA 310: 398-407.
3. Roush SW, Murphy TV (2007) Vaccine-Preventable Disease Table Working Group. Historical comparisons of morbidity and mortality for vaccine-preventable diseases in the United States. JAMA 298: 2155-2163.

4. Lopez A, Miranda P, Tejada E, Fishbein DB (1992) Outbreak of human rabies in the Peruvian jungle. Lancet 339: 408-411.

5. Williamson J, Ramirez R, Wingfield $\mathrm{T}$ (2015) Health, healthcare access, and use of traditional versus modern medicine in remote PeruvianAmazon communities: a descriptive study of knowledge, attitudes, and practices. Am J Trop Med Hyg 92: 857-864.

6. Stoner-Duncan B, Streicker DG2, Tedeschi CM (2014) Vampire bats and rabies: toward an ecological solution to a public health problem. PLoS Negl Trop Dis 8: e2867.

7. Streicker DG, Recuenco S, Valderrama W, Gomez Benavides J, Vargas I, et al. (2012) Ecological and anthropogenic drivers of rabies exposure in vampire bats: implications for transmission and control. Proc Biol Sci 279: 3384-3392.

8. Kessels JA, Recuenco S, Navarro-Vela AM, Deray R, Vigilato M, et al. (2017) Pre-exposure rabies prophylaxis: a systematic review. Bull World Health Organ 95: 210-219C.

9. Chulasugandha P, Khawplod P, Havanond P, Wilde H (2006) Cost comparison of rabies pre-exposure vaccination with post-exposure treatment in Thai children. Vaccine 24: 1478-1482.

10. Liu Q, Ertl HC (2012) Preventative childhood vaccination to rabies. Expert Opin Biol Ther 12: 1067-1075.

11. Liu X, Yang Y, Sun Z, Chen J, Ai J, et al. (2014) A recombinant rabies virus encoding two copies of the glycoprotein gene confers protection in dogs against a virulent challenge. PLoS One 9: e87105.

12. Xiang Z, Li Y, Cun A, Yang W, Ellenberg S, et al. (2006) Chimpanzee adenovirus antibodies in humans, sub-Saharan Africa. Emerg Infect Dis 12: 1596-1599.

13. Xiang ZQ, Greenberg L, Ertl HC, Rupprecht CE (2014) Protection of non-human primates against rabies with an adenovirus recombinant vaccine. Virology 451: 243-249.

14. Vellinga J, Smith JP, Lipiec A, Majhen D, Lemckert A, et al. (2014) Challenges in manufacturing adenoviral vectors for global vaccine product deployment. Hum Gene Ther 25: 318-327. 